\title{
A possible testbed for warped extra dimension from the angle of Buchdahl's limit
}

\author{
Tanmoy Paul ${ }^{\mathrm{a}}$ \\ Department of Theoretical Physics, Indian Association for the Cultivation of Science, 2A \& 2B Raja S.C. Mullick Road, Kolkata 700 032, India
}

Received: 17 November 2017 / Accepted: 13 June 2018 / Published online: 19 June 2018

(C) The Author(s) 2018

\begin{abstract}
We consider a five dimensional AdS warped spacetime in presence of a massive scalar field in the bulk. The scalar field potential fulfills the requirement of modulus stabilization even when the effect of backreaction of the stabilizing field is taken into account. In such a scenario, we investigate the possible role of modulus field on a compact stellar structure from the perspective of four dimensional effective theory. Our result reveals that in the presence of the modulus field, the upper bound of mass-radius ratio (generally known as Buchdahl's limit) of a star can go beyond the general relativity prediction. Interestingly this provides a natural testbed for the existence of such higher dimensional modulus field.
\end{abstract}

\section{Introduction}

Ever since the original proposal of Kaluza-Klein (KK) regarding the existence of extra spatial dimension(s) it is often believed that our universe is a 3-brane embedded in a higher dimensional spacetime and is described through a low energy effective theory on the brane carrying the signatures of extra dimensions [1,2]. Depending on different possible compactification schemes for the extra dimensions, a large number of models [3-15] have been constructed, and their predictions are yet to be observed in the current experiments.

Among various extra dimensional models proposed over the last several decades, Randall-Sundrum (RS) warped extra dimensional model [8] earned a special attention since it can resolve the gauge hierarchy problem without introducing any intermediate scale (between Planck and TeV) in the theory. In RS model the interbrane separation (known as modulus or radion) is assumed to be $\sim$ Planck length and generates the required hierarchy between the branes.
A suitable potential with a stable minimum is therefore needed for modulus stabilization. Goldberger and Wise (GW) proposed a useful stabilization mechanism [16] by introducing a massive scalar field in the bulk with appropriate boundary values. Though the backreaction of the stabilizing scalar field was originally neglected in GW proposal, its implications are subsequently studied in [17-19]. It has been demonstrated in [19] that the modulus of RS scenario can be stabilized using GW prescription even by incorporating the backreaction of the stabilizing field. Not only that, even the stable value of the modulus appears as a parameter in the low energy effective theory on the brane, but it's fluctuation about that stable value leads to dynamical modulus (or radion) field which couples to the fields on the observable brane. This attracted a large volume of work on phenomenological and cosmological implications [18,20-24] of modulus field in RS warped geometry model. This radion phenomenology along with the study of RS graviton [25-29] are considered to be the testing ground of warped extra dimensional models in collider experiments [30,31].

Apart from phenomenological setup, here we are interested to provide a possible testing ground for the existence of warped extra dimension from the angle of stable stellar structure namely the Buchdahl's limit.

There have been considerable interest in the compactness limit of any stellar structure, which, originally initiated by Buchdahl, indicates that under reasonable assumptions the minimum radius of a star has to be greater than (9/8) of its Schwarzschild radius [32-34]. These assumptions involve nature of the density of the star, which has to be decreasing outwards and also the interior solution has to be matched with an exterior one. This raises an intriguing question, how is the above limit modified if one considers a theory of gravity different from general relativity. This resulted into a large number of work quite extensively in recent times [35-44] (see also [45-49]).

\footnotetext{
a e-mail: pul.tnmy9@gmail.com
} 
The important questions that remain are:

1. How does the compactness limit (known as Buchdahl's limit) of a stellar structure modify due to the presence of radion field which carries the footprint of compactified warped extra dimension on our visible universe?

2. Can the modified Buchdahl's limit be a possible testing ground for such compactified extra dimension?

We address these questions in the present paper from the perspective of four dimensional effective theory.

Our paper is organized as follows. In Sect. 2, we describe the model. In Sect. 3 we find the possible modifications of Buchdahl's limit and its implications are discussed. In Sect. 4 , the interior spacetime of the stellar object is matched with a suitable exterior one. Finally we end the paper with some conclusive remarks.

\section{The model}

We consider a five dimensional AdS spacetime involving one warped and compact extra spacelike dimension. The spacetime is $S^{1} / Z_{2}$ orbifolded along the extra dimensional angular coordinate $\phi$, where the fixed points $\phi=(0, \pi)$ are identified with two 3-branes (3+1 dimensional), known as Planck (or hidden), $\mathrm{TeV}$ (or visible) brane respectively. Our usual four dimensional universe is the $\mathrm{TeV}$ brane and emerges as $4 \mathrm{D}$ effective theory. The opposite brane tensions along with the finely tuned five dimensional cosmological constant serve as energy-momentum tensor of the aforementioned configuration.

In higher dimensional braneworld scenario, the stabilization of extra dimensional modulus is a crucial aspect and needs to be addressed carefully. It has been demonstrated by Goldberger and Wise that the modulus corresponding to the radius of the extra dimension in warped geometry models can be stabilized [16] by invoking a massive scalar field in the bulk with non zero value on the branes.

Keeping the stabilization mechanism in mind, the braneworld setup considered in the present context is represented by the following action:

$$
\begin{aligned}
S= & \int d^{5} x \sqrt{-G}\left[M^{3} R-\Lambda-(1 / 2) G^{M N} \partial_{M} \Phi \partial_{N} \Phi-V(\Phi)\right] \\
& -\int d^{5} x \sqrt{-G}\left[\lambda_{\text {hid }} \delta(\phi)+\lambda_{\text {vis }} \delta(\phi-\pi)\right]
\end{aligned}
$$

where $M$ is the five dimensional Planck scale, $G_{M N}$ is the five dimensional metric. $\Lambda$ symbolizes the bulk cosmological constant, $\Phi$ is the scalar field endowed with a potential $V(\Phi)$, $\lambda_{\text {hid }}, \lambda_{\text {vis }}$ are the self interactions of scalar field (including brane tensions) on Planck, TeV branes.
We consider the metric ansatz as follows,

$d s^{2}=\exp [-2 A(\phi)] \eta_{\mu \nu} d x^{\mu} d x^{\nu}+r_{c}^{2} d \phi^{2}$

where $r_{c}$ is the compactification radius and $A(\phi)$ is termed as warp factor. For simplicity we assume that the bulk scalar field depends only on the extra dimensional coordinate $(\phi)$. Thus the 5-dimensional Einstein's and scalar field equations for this metric can be written as,

$$
\begin{aligned}
& \frac{4}{r_{c}^{2}} A^{\prime 2}(\phi)-\frac{1}{r_{c}^{2}} A^{\prime \prime}(\phi)=-\left(2 \kappa^{2} / 3\right) V(\Phi), \\
& -\left(\kappa^{2} / 3\right) \sum \lambda_{i}(\Phi) \delta\left(\phi-\phi_{i}\right), \\
& \frac{1}{r_{c}^{2}} A^{\prime 2}(\phi)=\frac{\kappa^{2}}{12 r_{c}^{2}} \Phi^{\prime 2}-\left(\kappa^{2} / 6\right) V(\Phi) \\
& \frac{1}{r_{c}^{2}} \Phi^{\prime \prime}(\phi)=\frac{4}{r_{c}^{2}} A^{\prime} \Phi^{\prime}+\frac{\partial V}{\partial \Phi}+\sum \frac{\partial \lambda_{i}}{\partial \Phi} \delta\left(\phi-\phi_{i}\right),
\end{aligned}
$$

where $M^{3}=\left(1 / 2 \kappa^{2}\right)$. Here index $i$ is used to designate the two branes and prime denotes the derivative with respect to $\phi$. From the above equations, the boundary conditions of $A(\phi)$ and $\Phi(\phi)$ are obtained as,

$$
\frac{1}{r_{c}}\left[A^{\prime}(\phi)\right]_{i}=\left(\kappa^{2} / 3\right) \lambda_{i}\left(\Phi_{i}\right)
$$

and

$$
\frac{1}{r_{c}}\left[\Phi^{\prime}(\phi)\right]_{i}=\partial_{\Phi} \lambda_{i}\left(\Phi_{i}\right) .
$$

Square brackets in the above two equations represent the jump of the corresponding variables on the branes. In order to get an analytic solution, let us consider the form of the scalar field potential as [18],

$V(\Phi)=(1 / 2) \Phi^{2}\left(u^{2}+4 u k\right)-\left(\kappa^{2} / 6\right) u^{2} \Phi^{4}$

where $k=\sqrt{-\kappa^{2} \Lambda / 6}$. The potential contains quadratic as well as quartic self interaction of the scalar field. Moreover it may be noticed that the mass and quartic coupling of the field $\Phi(\phi)$ are connected by a common free parameter $u$. Using this form of the potential, one obtains a solution of $A(\phi)$ and $\Phi(\phi)$ as follows,

$A(\phi)=k r_{c}|\phi|+\left(\kappa^{2} / 12\right) \Phi_{P}^{2} \exp \left(-2 u r_{c}|\phi|\right)$

and

$\Phi(\phi)=\Phi_{P} \exp \left(-u r_{c}|\phi|\right)$,

where $\Phi_{P}$ is taken as the value of the scalar field on the Planck brane. Using these solutions, $\lambda_{\text {hid }}$ and $\lambda_{\text {vis }}$ can be obtained from the boundary conditions (Eqs. (6), (7)) as,

$\lambda_{\text {hid }}=6 k / \kappa^{2}-u \Phi_{P}^{2}$ 
and

$\lambda_{v i s}=-6 k / \kappa^{2}+u \Phi_{P}^{2} \exp \left(-2 u \pi r_{c}\right)$.

In order to introduce the radion field, we consider a fluctuation of the inter-brane separation around the stable configuration $r_{c}$ [20]. This fluctuation can be treated as a field $T(x)$ (known as radion field) and for simplicity this new field is assumed to be the function of brane coordinates only. Then the metric takes the following form [20]:

$d s^{2}=\exp [-2 A(x, \phi)] g_{\mu \nu}(x) d x^{\mu} d x^{\nu}+T^{2}(x) d \phi^{2}$,

where $g_{\mu \nu}(x)$ is the induced on-brane metric and $A(x, \phi)$ has the following form,

$A(x, \phi)=k \phi T(x)+\frac{\kappa^{2} \Phi_{P}^{2}}{12} \exp [-2 u \phi T(x)]$.

Consequently $\Phi(x, \phi)$ can be obtained from Eq. (10) by replacing $r_{c}$ by $T(x)$ i.e.

$\Phi(x, \phi)=\Phi_{P} \exp (-u T(x) \phi)$

Plugging back the solutions presented in Eqs. (14), (15) into original five dimensional action (in Eq. (1)) and integrating over $\phi$ yields the four dimensional effective action as follows

$S_{\text {eff }}=\int d^{4} x \sqrt{-g}\left[\frac{M^{3}}{k} R_{(4)}-\frac{1}{2} g^{\mu \nu} \partial_{\mu} \Psi \partial_{\nu} \Psi-U_{\text {rad }}(\Psi)\right]$

where $R_{(4)}$ is the Ricci scalar formed by $g_{\mu \nu}(x)$. Moreover, $\Psi(x)=\sqrt{\frac{24 M^{3}}{k}} e^{-A(x, \pi)}[($ with $A(x, \pi)$ given in Eq. (14)] is the canonical radion field and $U_{\mathrm{rad}}(\Psi)$ is the radion potential with the following form [19]

$$
\begin{aligned}
U_{r a d}(\Psi)= & u^{2} \Phi_{P}^{2}\left[\frac{1}{u}\left(1-e^{-(2 u+4 k) \pi T(\Psi)}\right)\right. \\
& \left.-\frac{\kappa^{2} \Phi_{P}^{2}}{8(u+k)}\left(1-e^{-(4 u+4 k) \pi T(\Psi)}\right)\right],
\end{aligned}
$$

where $\kappa \Phi_{P}\left(=\frac{\Phi_{P}}{M^{3 / 2}}\right)$ is taken to be less than unity in order to ensure to validity of the classical solution. Moreover, $T(\Psi)$ is given by the expression: $\Psi(x)=$ $\sqrt{\frac{24 M^{3}}{k}} \exp \left[-k \pi T(x)-\frac{\kappa^{2} \Phi_{P}^{2}}{12} e^{-2 u \pi T(x)}\right]$, as defined earlier. Using this relation between $T(x)$ and $\Psi(x)$, we obtain the minimum of the radion potential $U_{\mathrm{rad}}(\Psi)$ at,

$$
\langle\Psi\rangle=\sqrt{\frac{24 M^{3}}{k}}\left(\frac{2 \sqrt{1+\frac{2 k}{u}}}{\kappa \Phi_{P}}\right)^{k / u} \exp \left[-\frac{1}{3}\left(1+\frac{2 k}{u}\right)\right],
$$

Further the mass squared $\left(m_{r a d}^{2}\right)$ of the radion field comes with the following expression

$$
m_{\text {rad }}^{2}=\frac{2 k^{2}}{3} e^{-2 k \pi\langle T\rangle}\left(\frac{u}{k}\right)^{3}\left[\frac{(1+2 k / u)}{\frac{1}{3}(1+2 k / u)(1+2 u / k)-1}\right]^{2}
$$

However our entire analysis of finding the stabilization condition in Eq. (18) is valid only for $u>0$. In this context one can easily check that the radion potential produces no minima for $u<0$. Hence the parameter $u$ is confined in positive regime in order to make a stable configuration for this braneworld scenario. For $\frac{u}{k} \lesssim 1$, the mass of the radion field becomes of $\mathrm{TeV}$ order $[18,20]$.

On projecting the bulk gravity on the brane, the extra degrees of freedom of $R^{(5)}$ (with respect to $R_{4}$ ) appears as a scalar field (the radion field), symbolized by $\Psi(x)$ in the four dimensional effective action (see Eq. (16)). For such onbrane theory, we are interested to explore the effect of radion field on stellar structure. Thus we further consider an extra matter density ( $L_{\text {mat }}$, confined on the brane) which acts as the ingredients of the star. Taking $L_{\text {mat }}$ into account, the final form of $4 \mathrm{D}$ effective action is as follows,

$$
\begin{aligned}
A_{e f f}= & S_{e f f}+\int d^{4} x \sqrt{-g} L_{m a t} \\
= & \int d^{4} x \sqrt{-g}\left[\frac{M^{3}}{k} R_{(4)}-\frac{1}{2} g^{\mu \nu} \partial_{\mu} \Psi \partial_{\nu} \Psi\right. \\
& \left.-U_{\text {rad }}(\Psi)+L_{\text {mat }}\right] .
\end{aligned}
$$

Therefore the radion field $\Psi$ (originated from extra dimension) and $L_{\text {mat }}$ serve as energy-momentum tensor in the four dimensional effective action. As the stellar interior is concerned, $L_{m a t}$ is taken to be a perfect fluid with energy-momentum tensor given by $T_{\nu}^{\mu}$ (matter) = $\operatorname{diag}(-\rho, p, p, p)$. Moreover the radion field contributes as,

$$
T_{\mu \nu}(\Psi)=\partial_{\mu} \Psi \partial_{\nu} \Psi-g_{\mu \nu}\left[\frac{1}{2} g^{\mu \nu} \partial_{\mu} \Psi \partial_{\nu} \Psi+U_{\text {rad }}(\Psi)\right] \text {. }
$$

This completes our preliminary discussion and provides the necessary steps that we will require in the next section while discussing the effect of radion field on a stellar structure from the perspective of effective four dimensional theory [described by Eq. (20)].

\section{Buchdahl's limit on stellar structure in presence of radion field}

As mentioned earlier, we want to investigate the possible modifications of Buchdahl's limit due to the presence of radion field, thus the spacetime that fit our purpose is static 
and a spherically symmetric. Therefore the metric ansatz for the interior star is taken as,

$$
\begin{aligned}
d s_{-}^{2} & =g_{\mu \nu} d x^{\mu} d x^{\nu} \\
& =-e^{\nu(r)} d t^{2}+e^{\lambda(r)} d r^{2}+r^{2}\left(d \theta^{2}+\sin ^{2} \theta d \phi^{2}\right),
\end{aligned}
$$

where $\nu(r)$ and $\lambda(r)$ are arbitrary functions of radial coordinate $r$ that we need to determine from gravitational equations. Such spherically symmetric spacetime ensures that $\Psi$ as well as $\rho$ and $p$ are the functions of $r$ only. Hence Eq. (21) can be simplified and as a consequence the energy-momentum tensors of matter field, radion field are given by

$$
\begin{aligned}
T_{v}^{\mu}(\text { matter })= & \operatorname{diag}(-\rho(r), p(r), p(r), p(r)), \\
T_{v}^{\mu}(\Psi)= & \operatorname{diag}\left(-f^{2}(r)-U_{\text {rad }}(r), f^{2}(r)-U_{\text {rad }}(r),\right. \\
& \left.-f^{2}(r)-U_{\text {rad }}(r),-f^{2}(r)-U_{\text {rad }}(r)\right)
\end{aligned}
$$

respectively, where $f^{2}(r)$ is defined as $f^{2}(r)=\frac{1}{2} e^{-\lambda(r)}$ $\Psi^{\prime}(r)^{2}$ and $U_{\text {rad }}(r)=U_{\text {rad }}(\Psi(r))$. Considering the interior of the stellar object to be filled with perfect fluid having energy-momentum tensor presented in Eq. (23), the gravitational equations [for the metric ansatz mentioned in Eq. (22)] become,

$$
\begin{aligned}
& e^{-\lambda}\left[\frac{1}{r^{2}}-\frac{\lambda^{\prime}}{r}\right]-\frac{1}{r^{2}}=8 \pi G_{4}\left[-\rho-f^{2}-U_{r a d}(r)\right], \\
& e^{-\lambda}\left[\frac{v^{\prime}}{r}+\frac{1}{r^{2}}\right]-\frac{1}{r^{2}}=8 \pi G_{4}\left[p+f^{2}-U_{r a d}(r)\right],
\end{aligned}
$$

where ' denotes the derivative with respect to $r$ and $\frac{1}{8 \pi G_{4}}=$ $\frac{M^{3}}{k} \sim 10^{38}(\mathrm{GeV})^{2}$. There exists another Einstein's equation corresponding to angular coordinate, but that can be derived from the above two and hence is not independent.

On the other hand, the conservation equation for the fluid and the field equation for radion field takes the following simple form in the context of spherically symmetric spacetime,

$p^{\prime}+\frac{v^{\prime}}{2}(\rho+p)=0$

and

$f^{\prime}+\frac{v^{\prime}}{2} f+\frac{2}{r} f=\frac{U_{r a d}^{\prime}}{2 f}$

respectively. To derive the radion field equation, we use the definition of $f^{2}(r)$ as mentioned earlier. At this stage, it deserves mentioning that there are four independent differential equations governing the behaviour of the system considered in the present case, while there are five unknowns, $\lambda(r)$, $v(r), \rho(r), p(r), f(r)$. This problem is generally resolved by assuming an equation of state for the perfect fluid. However this equation of state is not needed in the present context, because here we are interested on the upper bound of the mass-radius ratio of the star (in presence of modulus field), for which the complete interior solutions are not necessary

Next we try to get some information about the functions $\lambda(r), v(r), \Psi(r)$ from the above equations of motion. It is easy to show that one can integrate Eq. (25), resulting into the following form of $\lambda(r)$,

$$
\begin{aligned}
e^{-\lambda(r)} & =1-\frac{2 G_{4}}{r} \int_{0}^{r} 4 \pi r^{2}\left[\rho+f^{2}+U_{\text {rad }}(r)\right] \\
& =1-\frac{2 G_{4} m(r)}{r}
\end{aligned}
$$

where $m(r)=\int_{0}^{r} 4 \pi r^{2}\left[\rho+f^{2}+U_{\text {rad }}(r)\right]$, the mass of the star up to radius $r$. It is clear from the expression of $m(r)$ that due to the presence of radion field, the total gravitational mass is different from that the actual matter density present inside. The extra gravitating mass comes from the radion field strength and its potential.

However, Eq. (27) can be rewritten as,

$v^{\prime}(r)=\frac{-2 p^{\prime}}{(\rho+p)}$

which is the famous Tolman-Oppenheimer-Volkoff (TOV) equation. The matter density $(\rho)$ as well as the pressure $(p)$ inside the star generally decreases with an increase of $r$. This behaviour of $p$ along with Eq. (30) indicate that the function $v(r)$ increases with $r$ (i.e $v^{\prime}(r)>0$ ).

Considering $\xi$ as the fluctuation of the radion field about its vacuum expectation value i.e $\Psi=\langle\Psi\rangle+\xi$ (see Eq. (18) for the explicit expression of $\langle\Psi\rangle$ ), the potential term $U_{\text {rad }}$ can be expressed as,

$U_{\text {rad }}(\xi)=U_{\text {rad }}(\langle\Psi\rangle)+\frac{1}{2} m_{r a d}^{2} \xi^{2}$

where the terms up to quadratic order in $\xi$ are retained for $\xi \sqrt{8 \pi G_{4}}<1$. Further $U_{\text {rad }}(\langle\Psi\rangle)=u \Phi_{P}^{2}$ (recall $u>0$ in order to stabilize the inter-brane separation) and $m_{\text {rad }}^{2}$ is the radion mass squared, given in Eq. (19). With the above expression of $U_{\text {rad }}(\xi)$, the equation of motion for $\xi(r)$ (see Eq. (28)) takes the following form:

$$
\begin{gathered}
\xi^{\prime \prime}(r)+\left[\frac{2}{r}+\frac{\nu^{\prime}(r)-\lambda^{\prime}(r)}{2}\right] \xi^{\prime}(r) \\
-m_{r a d}^{2} e^{\lambda} \xi(r)=0
\end{gathered}
$$

Here we are interested on the solution of $\xi(r)$ in the region - just inside the surface of the star i.e. for $r=r_{0}(1-\epsilon)$ ( $0<\epsilon<1$ and $r_{0}$ is the radius of the star). In this regime of $r$, we retain the terms up to linear order in $\epsilon$ and thus Eq. (32) turns out to be,

$$
\begin{aligned}
& \frac{d}{d \epsilon}\left[\frac{d \xi}{d \epsilon}\right]-\left[2+\frac{\left(v_{0}^{\prime}-\lambda_{0}^{\prime}\right) r_{0}}{2}+2 \epsilon\right] \frac{d \xi}{d \epsilon} \\
& -m_{r a d}^{2} e^{\lambda_{0}} r_{0}^{2} \xi(\epsilon)=0
\end{aligned}
$$


where we use $\xi^{\prime}(r)=-\frac{1}{r_{0}} \frac{d \xi}{d \epsilon}$ and $\xi^{\prime \prime}(r)=\frac{1}{r_{0}^{2}} \frac{d^{2} \xi}{d \epsilon^{2}}$. Moreover in the vicinity of $r=r_{0}$, the functions $v(r), \lambda(r)$ along with their derivatives are approximated as their values at $r=$ $r_{0}$ (the subscripts ' 0 ' in Eq. (33) denote the values of the respective functions at $r=r_{0}$, as for example $v_{0}=v\left(r_{0}\right)$ ). We solve Eq. (33) and the solution is given by,

$$
\begin{aligned}
& \xi(\epsilon)=\exp \left[-\left(2+\frac{\left(v_{0}^{\prime}-\lambda_{0}^{\prime}\right) r_{0}}{2}\right) \epsilon-\epsilon^{2}\right] \\
& \quad \times \text { Hypergeometric } 1 F 1\left(1+\frac{m_{r a d}^{2} r_{0}^{2} e^{\lambda_{0}}}{2}, \frac{1}{2},\left(2+\frac{\left(v_{0}^{\prime}-\lambda_{0}^{\prime}\right) r_{0}}{2}\right) \epsilon\right)
\end{aligned}
$$

Retaining up to the linear order terms in $\epsilon$, Eq. (34) lands with the following form

$$
\xi(\epsilon)=1+\left(1+\frac{m_{r a d}^{2} r_{0}^{2} e^{\lambda_{0}}}{2}\right)\left(2+\frac{\left(v_{0}^{\prime}-\lambda_{0}^{\prime}\right) r_{0}}{2}\right) \epsilon
$$

The above solution of $\xi(\epsilon)$ immediately leads to the following expression of $\frac{d}{d r}\left[\xi^{2}(r)\right]$ in the limit $r \rightarrow r_{0}^{-}$(i.e just inside the surface of the star):

$\left.\frac{d}{d r}\left[\xi^{2}(r)\right]\right|_{r \rightarrow r_{0}^{-}}=\left(1+\frac{m_{r a d}^{2} r_{0}^{2} e^{\lambda_{0}}}{2}\right)\left(2+\frac{\left(v_{0}^{\prime}-\lambda_{0}^{\prime}\right) r_{0}}{2}\right)$

However using Eq. (35) along with the gravitational equations presented in Eqs. (25) and (26), we obtain

$$
\begin{aligned}
{\left[2+\frac{\left(v_{0}^{\prime}-\lambda_{0}^{\prime}\right) r_{0}}{2}\right]=} & e^{\lambda_{0}}\left[1-\frac{1}{2} m_{r a d}^{2} r_{0}^{2}\right] \\
& +1-4 \pi G e^{\lambda_{0}} \rho_{0} r_{0}^{2}-2 u \Phi_{P}^{2}
\end{aligned}
$$

To derive Eq. (37), we use the fact the pressure of the fluid is zero on the surface of the star i.e $p\left(r_{0}\right)=0$.

As demonstrated in Sect. 2, the mass of the radion field is of $\mathrm{TeV}$ order i.e $m_{\text {rad }} \sim 10^{3} \mathrm{GeV}$. Further the radius of a compact star typically comes as $r_{0} \sim 10^{18}(\mathrm{GeV})^{-1}$ (for neutron star). With these numerical values and due to the condition $e^{\lambda_{0}}>1$ (see Eq. (29)), it is clear that the right hand side of Eq. (37) is negative and thus one can write

$\left[2+\frac{\left(v_{0}^{\prime}-\lambda_{0}^{\prime}\right) r_{0}}{2}\right]<0$

As a result, Eq. (36) leads to the following inequality

$\left.\frac{d}{d r}\left[\xi^{2}(r)\right]\right|_{r \rightarrow r_{0}^{-}}<0$

Equation (39) indicates that $\xi^{2}(r)$ has a negative gradient in the inward vicinity of $r=r_{0}$. Therefore by considering the monotonic behaviour of the radion field inside the star, it can be argued that the negative gradient of $\xi^{2}(r)$ holds for $r<r_{0}$ i.e

$\left.\frac{d}{d r}\left[\xi^{2}(r)\right]\right|_{r<r_{0}}<0$ which clearly implies that $U_{\text {rad }}^{\prime}\left(r<r_{0}\right)=\frac{1}{2} m_{\text {rad }}^{2} \frac{d}{d r}\left[\xi^{2}(r)\right]$ $\left.\right|_{r<r_{0}}<0$.

However Eq. (28) can be expressed as $\frac{d \rho_{\text {rad }}}{d r}=-v^{\prime}(r) f^{2}-$ $\frac{4}{r} f^{2}+2 U_{\text {rad }}^{\prime}(r)$, where $\rho_{\text {rad }}\left(=f^{2}(r)+U_{\text {rad }}\right)$ denotes the energy density of the radion field. Thereby the condition $U_{\text {rad }}^{\prime}<0$ and due to the fact $\nu^{\prime}(r)>0$ (see Eq. (30)), the above expression of $\rho_{\text {rad }}^{\prime}$ clearly implies that $\rho_{\text {rad }}$ decreases with the radial coordinate $r$. Hence the effective energy density (inside the star) $\rho+f^{2}+U_{\text {rad }}$ also decreases as the surface of the star is approached. We will use this result later on.

With these ingredients, let us now derive Buchdahl's limit explicitly and for that let us start by differentiating both sides of Eq. (26) (with respect to $r$ ) and get,

$$
\begin{aligned}
& e^{-\lambda}\left[\frac{v^{\prime \prime}}{r}-\frac{v^{\prime}}{r^{2}}-\frac{2}{r^{3}}-\frac{\lambda^{\prime} v^{\prime}}{r}-\frac{\lambda^{\prime}}{r^{2}}\right]+\frac{2}{r^{3}}=8 \pi G_{4} \\
& \quad \times\left[p^{\prime}+2 f f^{\prime}-U_{r a d}^{\prime}(r)\right] .
\end{aligned}
$$

Using the conservation equations for the fluid and the radion field from Eqs. (27) and (28), one can evaluate the right hand side of Eq. (41), leading to

$$
\begin{aligned}
& 8 \pi G_{4}\left[p^{\prime}+2 f f^{\prime}-U_{r a d}^{\prime}(r)\right] \\
& =8 \pi G_{4}\left[-\frac{v^{\prime}}{2}(\rho+p)+2 f\left(-\frac{v^{\prime}}{2} f-\frac{2}{r} f\right)\right] \\
& =e^{-\lambda}\left[-\frac{v^{\prime 2}}{2 r}-\frac{v^{\prime} \lambda^{\prime}}{2 r}\right]-\frac{32 \pi G_{4}}{r} f^{2}
\end{aligned}
$$

Substituting the above expression back to Eq. (41) and a little simplification leads to the following equation,

$$
\begin{aligned}
2 r v^{\prime \prime}+r v^{\prime 2}-r v^{\prime} \lambda^{\prime}-2 \nu^{\prime}= & \frac{4}{r}\left(1-e^{\lambda}\right)+2 \lambda^{\prime} \\
& -64 \pi G_{4} r f^{2} e^{\lambda}
\end{aligned}
$$

By using the following two identities namely

$\frac{d}{d r}\left[\frac{1}{r} e^{-\lambda / 2} \frac{d e^{\nu / 2}}{d r}\right]=\frac{e^{(\nu-\lambda) / 2}}{4 r^{2}}\left[2 r v^{\prime \prime}+r v^{\prime 2}-r v^{\prime} \lambda^{\prime}-2 \nu^{\prime}\right]$

$$
\frac{d}{d r}\left[\frac{1-e^{-\lambda}}{2 r^{2}}\right]=\frac{e^{-\lambda}}{2 r^{3}}\left[r \lambda^{\prime}-2\left(e^{\lambda}-1\right)\right]
$$

Equation (43) can be rewritten as follows,

$$
\begin{aligned}
e^{-(\nu+\lambda) / 2} \frac{d}{d r}\left[\frac{1}{r} e^{-\lambda / 2} \frac{d e^{\nu / 2}}{d r}\right]= & \frac{d}{d r}\left[\frac{1-e^{-\lambda}}{2 r^{2}}\right] \\
& -\frac{16 \pi G_{4}}{r} f^{2}
\end{aligned}
$$

At this point, we put forward some sensible requirements: the average energy density inside the star $\rho_{a v}=m(r) / r^{3}$ should decrease with the radial coordinate. Even though the average density involves contribution from the radion field, 
since the radion field strength itself decreases outwards, the above requirement will trivially hold. Further, the form of $e^{-\lambda}$ given in Eq. (29) indicates that the first term of the right hand side of Eq. (46) is essentially $d \rho_{a v} / d r$ i.e. $\frac{d}{d r}\left[\frac{1-e^{-\lambda}}{2 r^{2}}\right]=$ $\frac{d \rho_{a v}}{d r}$. This expression along with the decreasing character of $\rho_{a v}$ (with $r$ ) makes the right hand side of Eq. (46) negative. As a consequence, we get the following inequality:

$\frac{d}{d r}\left[\frac{1}{r} e^{-\lambda / 2} \frac{d e^{\nu / 2}}{d r}\right]<0$.

Integrating the above relation from some radius $r$ within the star to the surface of the star, given by the radius $r_{0}$, we obtain,

$\frac{d e^{\nu / 2}}{d r}>\frac{v_{0}^{\prime}}{2 r_{0}} r e^{\lambda / 2} e^{\left(\nu_{0}-\lambda_{0}\right) / 2}$,

where the quantities with the subscript ' 0 ' denotes that they are to be evaluated at the surface of the star i.e. at $r=r_{0}$. Furthermore, to derive the above inequality, we consider that both the metric and its first derivative are continuous at $r=$ $r_{0}$. However, later, in Sect. 4, we explicitly find the continuity conditions of metric and its first derivative at the boundary of the star by considering a generalized Vaidya metric for the exterior spacetime of the stellar object.

Integrating again the both sides of Eq. (48) from the origin to the surface of the star, we obtain

$$
\begin{gathered}
e^{\nu_{0} / 2}-e^{\nu / 2}(r=0)>\frac{\nu_{0}^{\prime}}{2 r_{0}} e^{\left(\nu_{0}-\lambda_{0}\right) / 2} \int_{0}^{r_{0}} r e^{\lambda / 2} d r \\
=\frac{v_{0}^{\prime}}{2 r_{0}} e^{\left(\nu_{0}-\lambda_{0}\right) / 2} \int_{0}^{r_{0}} d r \frac{r}{\sqrt{1-\frac{2 G_{4} m(r)}{r}}} .
\end{gathered}
$$

In the last line, we use the solution of $e^{\lambda}$ (see Eq. (29)). As the average energy density decreases towards the boundary of the star, it immediately follows that $m(r) / r^{3}>M / r^{3}$, where $M=m\left(r_{0}\right)$, the total effective mass of the star. Thus the inequality in Eq. (49) holds more strongly if $\frac{m(r)}{r}$ is replaced by $\frac{M}{r_{0}^{3}} r^{2}$. With this modification, we arrive at,

$$
\begin{gathered}
e^{\nu_{0} / 2}-e^{\nu / 2}(r=0)>\frac{v_{0}^{\prime}}{2 r_{0}} e^{\left(\nu_{0}-\lambda_{0}\right) / 2} \int_{0}^{r_{0}} d r \frac{r}{\sqrt{1-\frac{2 G_{4} M}{r_{0}^{3}} r^{2}}} \\
=\frac{v_{0}^{\prime}}{2 r_{0}} e^{\left(\nu_{0}-\lambda_{0}\right) / 2}\left(\frac{r_{0}^{3}}{2 G_{4} M}\right)\left[1-\sqrt{1-\frac{2 G_{4} M}{r_{0}}}\right] .
\end{gathered}
$$

Both the pressure and the contribution of the radion field are positive and finite at the origin, it follows that $e^{v / 2}(r=$ $0)>0$. Applying this result into Eq. (50), we immediately obtain the following inequality,

$1-\frac{v_{0}^{\prime}}{2 r_{0}} e^{-\lambda_{0} / 2}\left(\frac{r_{0}^{3}}{2 G_{4} M}\right)\left[1-\sqrt{1-\frac{2 G_{4} M}{r_{0}}}\right]>0$.
The factor $\nu_{0}^{\prime}$ can be obtained in terms of $\lambda_{0}$ by considering Eq. (26) at the surface of the star $\left(r=r_{0}\right.$, where the pressure is zero i.e. $\left.p\left(r_{0}\right)=0\right)$ as,

$\frac{\nu_{0}^{\prime}}{2 r_{0}}=-\frac{1}{2 r_{0}^{2}}+\frac{1}{2} e^{\lambda_{0}}\left[\frac{1}{r_{0}^{2}}+8 \pi G_{4}\left(f_{0}^{2}-U_{\text {rad }}^{0}\right)\right]$.

Recall $f_{0}^{2}=\frac{1}{2} e^{-\lambda_{0}} \Psi^{\prime}\left(r_{0}\right)^{2}$ and $U_{r a d}^{0}$ is the radion potential at $r_{0}$. Plugging the above expression of $v_{0}^{\prime}$ into Eq. (51) and further using the solution of $e^{\lambda}$, we finally lands up with the inequality as follows:

$$
\begin{aligned}
& \sqrt{1-\frac{2 G_{4} M}{r_{0}}-\frac{r_{0}^{3}}{2 G_{4} M}\left(1-\sqrt{1-\frac{2 G_{4} M}{r_{0}}}\right)} \\
& \times\left[\frac{1}{2 r_{0}^{2}}\left(\frac{2 G_{4} M}{r_{0}}\right)+4 \pi G_{4}\left(f_{0}^{2}-U_{\text {rad }}^{0}\right)\right]>0 .
\end{aligned}
$$

Simplification of Eq. (53) yields a quadratic expression for $\frac{2 G_{4} M}{r_{0}}$, one root of which corresponds to a negative value and hence can be safely ignored, while the other root provides the necessary limit on mass-radius ratio (i.e. $\frac{M}{r_{0}}$ ) of the star, as follows,

$$
\frac{2 G_{4} M}{r_{0}}<\frac{b}{2 a}\left[1+\sqrt{1+\frac{4 a c}{b^{2}}}\right]
$$

where $a, b, c$ have the following expressions:

$$
\begin{aligned}
a= & \frac{9}{4}-6 \pi G_{4} r_{0}^{2} \Psi_{0}^{\prime 2}+4 \pi^{2} G_{4}^{2} r_{0}^{4} \Psi_{0}^{\prime 4}, \\
b= & 2-10 \pi G_{4} r_{0}^{2} \Psi_{0}^{\prime 2}+8 \pi^{2} G_{4}^{2} r_{0}^{4} \Psi_{0}^{\prime 4} \\
& +12 \pi G_{4} r_{0}^{2} U_{r a d}^{0}-16 \pi^{2} G_{4}^{2} r_{0}^{4} \Psi_{0}^{\prime 2} U_{r a d}^{0}, \\
c= & 4 \pi G_{4} r_{0}^{2} \Psi_{0}^{\prime 2}-4 \pi^{2} G_{4}^{2} r_{0}^{4} \Psi_{0}^{\prime 4}-8 \pi G_{4} r_{0}^{2} U_{r a d}^{0} \\
& -16 \pi^{2} G_{4}^{2} r_{0}^{4}\left(U_{\text {rad }}^{0}\right)^{2}+16 \pi^{2} G_{4}^{2} r_{0}^{4} \Psi_{0}^{\prime 2} U_{r a d}^{0} .
\end{aligned}
$$

Equations (55)-(57) indicate that in the absence of the radion field, $a, b, c$ take the value as $\frac{9}{4}, 2,0$ respectively, for which one immediately recovers the usual Buchdahl's limit in General Relativity, i.e $\frac{2 G_{4} M}{r_{0}}<\frac{8}{9}$.

However, in the presence of the radion field $(\Psi)$, the upper limit on mass-radius ratio of a stable stellar object gets modified compared to general relativity and obviously depends on the strength of the radion field (and its potential) on the surface of the star. From Eq. (54) (along with the expressions of $a, b, c$ ), we obtain Fig. 1 demonstrating the variation of $\frac{2 G_{4} M}{r_{0}}$ with $f_{0}$ and $U_{\text {rad }}^{0}$.

Figure 1 (with the contour plot) clearly reveals that in the presence of higher dimensional modulus field, the upper bound on $\frac{2 G_{4} M}{r_{0}}$ can be larger than $8 / 9$ and reach up to unity. Therefore extra mass can be packed into the stellar structure in comparison to the Einstein gravity. This provides an interesting testbed for existence of the modulus field (or radion field) which carries the footprint of compactified extra dimension on our visible universe. Thus, if a compact stellar object (say a neutron star) is observed whose $2 G_{4} M / r_{0}$ is larger 
Fig. $1 \frac{2 G_{4} M}{r_{0}}$ vs $G_{4} r_{0}^{2} \Psi_{0}^{\prime 2}(=x)$ and $G_{4} r_{0}^{2} U_{\text {rad }}^{0}(=y)$ (left figure), corresponding contour plot (right figure)

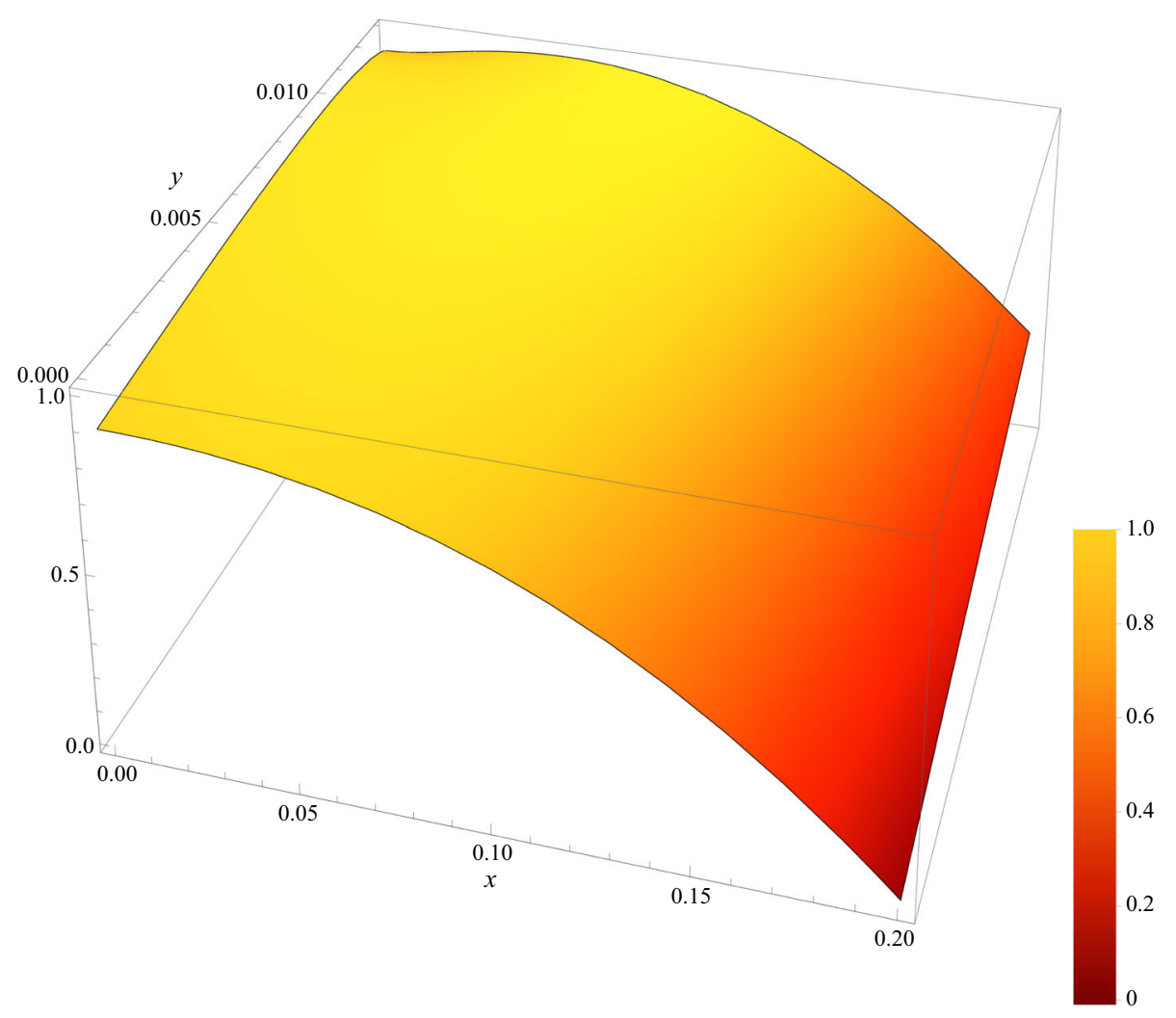

than $8 / 9$ (i.e $\frac{2 G_{4} M}{r_{0}}$ lies between $[8 / 9,1]$ ), then it can possibly signal towards the existence of such higher dimension.

\section{Matching of the interior spacetime with an exterior geometry}

To complete the model, the interior spacetime geometry of the spherical star needs to be matched to an exterior geometry. For the required matching, the Israel conditions are used, where the metric coefficients and extrinsic curvatures (first and second fundamental forms respectively) are matched at the boundary of the sphere.

However in the presence of a scalar field (which is the radion field in the present context), the interior spacetime can not be smoothly matched to a vacuum exterior (i.e. the Schwarzschild one). If the exterior is a vacuum, the scalar field has to behave as a delta function at the boundary resulting in a square of delta function for the energy density.

To avoid this problem, in the present case, we match the interior spacetime with a generalized Vaidya exterior spacetime at the boundary hypersurface $\Sigma$ given by $r=r_{0}$. The metric inside and outside of $\Sigma$ are given by,

$$
d s_{-}^{2}=-e^{\nu(r)} d t^{2}+e^{\lambda(r)} d r^{2}+r^{2}\left(d \theta^{2}+\sin ^{2} \theta d \phi^{2}\right)
$$

and

$$
\begin{aligned}
d s_{+}^{2}= & -\left(1-\frac{2 G_{4} M_{+}\left(r_{v}\right)}{r_{v}}\right) d v^{2}+2 d v d r_{v} \\
& +r_{v}^{2}\left(d \theta^{2}+\sin ^{2} \theta d \varphi^{2}\right)
\end{aligned}
$$

respectively, where $r_{v}, v, \theta$ and $\varphi$ are the exterior coordinates and $M_{+}\left(r_{v}\right)$ (the suffix ' + ' stands for exterior) is exterior mass function, which is independent of $v$ due to the reason that the spacetime is static. The same hypersurface $\Sigma$ can alternatively be defined by the exterior coordinates as $r_{v}=$ $R(t)$ and $v=T(t)$. Then the metrics on $\Sigma$ from inside and outside coordinates turn out to be,

$d s_{-, \Sigma}^{2}=-e^{\nu_{0}} d t^{2}+r_{0}^{2} d \Omega^{2}$

and

$d s_{+, \Sigma}^{2}=-\left[\left(1-\frac{2 G_{4} M_{+, \Sigma}}{R(t)}\right) \dot{T}^{2}-2 \dot{T} \dot{R}\right] d t^{2}+R(t)^{2} d \Omega^{2}$,

where $M_{+, \Sigma}$ is the exterior mass function on $\Sigma, d \Omega^{2}$ denotes the line element on a unit two sphere and dot represents $\frac{d}{d t}$. Matching the first fundamental form on $\Sigma$ (i.e. $d s_{-, \Sigma}^{2}=$ $d s_{+, \Sigma}^{2}$ ) yields the following two conditions:

$\frac{d T(t)}{d t}=\frac{e^{\nu_{0} / 2}}{\sqrt{1-\frac{2 G_{4} M_{+, \Sigma}}{r_{0}}}}$ 
and

$R(t)=r_{0}$

In order to match the second fundamental form, we calculate the normal of the hypersurface $\Sigma$ from inside $\left(\vec{n}_{-}=n_{-}^{t}, n_{-}^{r}\right.$, $\left.n_{-}^{\theta}, n_{-}^{\varphi}\right)$ and outside $\left(\vec{n}_{+}=n_{+}^{v}, n_{+}^{r_{v}}, n_{+}^{\theta}, n_{+}^{\varphi}\right)$ coordinates as follows,

$n_{-}^{t}=0, \quad n_{-}^{r}=e^{-\lambda_{0} / 2}, \quad n_{-}^{\theta}=n_{-}^{\varphi}=0$

and

$n_{+}^{v}=\frac{1}{\sqrt{1-\frac{2 G_{4} M_{+, \Sigma}}{r_{0}}}}$,

$n_{+}^{r_{v}}=\sqrt{1-\frac{2 G_{4} M_{+, \Sigma}}{r_{0}}}$,

$n_{+}^{\theta}=n_{+}^{\varphi}=0$.

To derive the normal vectors, we use Eq. (61). The above expressions of $\vec{n}_{-}$and $\vec{n}_{+}$leads to the extrinsic curvature of $\Sigma$ from interior and exterior coordinates respectively, and are given by,

$K_{t t}^{-}=-\frac{\nu_{0}^{\prime}}{2} e^{\nu_{0}} e^{-\lambda_{0} / 2}$,

$K_{\theta \theta}^{-}=r_{0} e^{-\lambda_{0} / 2}, \quad K_{\varphi \varphi}^{-}=r_{0} e^{-\lambda_{0} / 2} \sin ^{2} \theta$,

from interior metric, and

$$
\begin{aligned}
& K_{t t}^{+}=-G_{4} \frac{e^{\nu_{0}}}{\sqrt{1-2 \frac{G_{4} M_{+, \Sigma}}{r_{0}}}}\left[\frac{M_{+, \Sigma}}{r_{0}^{2}}-\left.\frac{1}{r_{0}} \frac{\partial M_{+}\left(r_{v}\right)}{\partial r_{v}}\right|_{\Sigma}\right], \\
& K_{\theta \theta}^{+}=r_{0} \sqrt{1-\frac{2 G_{4} M_{+, \Sigma}}{r_{0}}}, \\
& K_{\varphi \varphi}^{+}=r_{0} \sqrt{1-\frac{2 G_{4} M_{+, \Sigma}}{r_{0}}} \sin \theta^{2}
\end{aligned}
$$

from exterior metric.

The equality of the extrinsic curvatures of $\Sigma$ from both sides is therefore equivalent to the following two conditions:

$e^{\left.-\lambda_{0}\right) / 2}=\sqrt{1-\frac{2 G_{4} M_{+, \Sigma}}{r_{0}}}$

and

$\left.\frac{\partial M_{+}\left(r_{v}\right)}{\partial r_{v}}\right|_{\Sigma}=\frac{M_{+, \Sigma}}{r_{0}}-\frac{r_{0}}{2 G_{4}} v_{0}^{\prime}\left[1-\frac{2 G_{4} M_{+, \Sigma}}{r_{0}}\right]$.

The total mass of the spherical star is given by: $M=$ $\int_{0}^{r_{0}} 4 \pi r^{2}\left(\rho+f^{2}+U_{r a d}(r)\right)$. Equation (66) along with the solution of $\lambda$ (see Eq. (29)) relates the exterior mass function on $\Sigma$ (i.e. $M_{+, \Sigma}$ ) with the total mass of the stellar object as,

$$
\begin{aligned}
M_{+, \Sigma} & =M \\
& =\int_{0}^{r_{0}} 4 \pi r^{2}\left(\rho+f^{2}+U_{r a d}(r)\right) .
\end{aligned}
$$

Equations (60), (61), (67), (68) completely specify the matching at the boundary of the star with an exterior generalized Vaidya geometry.

\section{Conclusion}

We consider a five dimensional AdS compactified warped geometry model with two 3-branes embedded within the spacetime. For the purpose of modulus stabilization, a massive scalar field is invoked in the bulk and its backreaction on spacetime geometry is taken into account. In such a scenario, our universe is identified with a 3-brane and emerges as a four dimensional effective theory. On projecting the bulk gravity on the brane, the extra degrees of freedom of $R^{(5)}$ appears as a scalar field (known as radion field) in the 4D effective on-brane theory. From the perspective of such onbrane theory, we explore the effect of radion field on the limit of mass-radius ratio $\left(M / r_{0}\right)$ for a stable stellar structure.

We match the interior spacetime of the star with a suitable exterior geometry on the boundary $(\Sigma)$. For this matching, the Israel junction conditions are used where the metric coefficients and extrinsic curvatures are matched on $\Sigma$. At this stage, it deserves mention that in presence of a scalar field (which is the radion field in the present context), the matching of interior spacetime with exterior Schwarzschild geometry leads to some inconsistency. For instance, since Schwarzschild has zero scalar field, such a matching would lead to a discontinuity in the scalar field, which means a delta function in the gradient of the scalar field. As a consequence, there will appear square of a delta function in the stress-energy, which is definitely an inconsistency. To avoid such problems, here we consider the exterior geometry as a generalized Vaidya spacetime. With this consideration, we determine the matching conditions given in Eqs. (60), (61), (67), (68).

The main conclusion of the present investigation is the following. Due to the presence of radion field, the upper limit on mass-radius ratio (generally known as Buchdahl's limit) of a compact stellar object gets modified in comparison to general relativity and obviously depends on the strength of the radion field (and its potential) on the surface of the star. The variation of Buchdahl's limit with the radion field strength is shown in Fig. 1, which clearly demonstrates that in the presence of the higher dimensional modulus field, the upper bound of $\frac{2 G_{4} M}{r_{0}}$ can go beyond the value $8 / 9$ and reach up to unity; while the general relativity prediction is given by: $\frac{2 G_{4} M}{r_{0}}<\frac{8}{9}$. Therefore extra mass can be packed into the stellar structure in comparison to the Einstein gravity. This provides an interesting testbed for existence of modulus field (or radion field) which carries the footprint of compactified extra dimension on our visible universe. Hence if it is possible to detect a compact object with mass-radius ratio larger 
than the general relativity prediction, then one can infer about the possible presence of such higher dimension.

Acknowledgements The author would like to thank Narayan Banerjee and Soumitra SenGupta for illuminating discussions.

Open Access This article is distributed under the terms of the Creative Commons Attribution 4.0 International License (http://creativecomm ons.org/licenses/by/4.0/), which permits unrestricted use, distribution, and reproduction in any medium, provided you give appropriate credit to the original author(s) and the source, provide a link to the Creative Commons license, and indicate if changes were made.

Funded by SCOAP ${ }^{3}$.

\section{References}

1. S. Kanno, J. Soda, Phys. Rev. D 66, 083506 (2002)

2. T. Shiromizu, K. Maeda, M. Sasaki, Phys. Rev. D 62, 024012 (2000)

3. N. Arkani-Hamed, S. Dimopoulos, G. Dvali, Phys. Lett. B 429, 263 (1998)

4. N. Arkani-Hamed, S. Dimopoulos, G. Dvali, Phys. Rev. D 59, 086004 (1999)

5. I. Antoniadis, N. Arkani-Hamed, S. Dimopoulos, G. Dvali, Phys. Lett. B 436, 257 (1998)

6. P. Horava, E. Witten, Nucl. Phys. B 475, 94 (1996)

7. P. Horava, E. Witten, Nucl. Phys. B 460, 506 (1996)

8. L. Randall, R. Sundrum, Phys. Rev. Lett. 83, 3370 (1999)

9. N. Kaloper, Phys. Rev. D 60, 123506 (1999)

10. T. Nihei, Phys. Lett. B 465, 81 (1999)

11. H.B. Kim, H.D. Kim, Phys. Rev. D 61, 064003 (2000)

12. A.G. Cohen, D.B. Kaplan, Phys. Lett. B 470, 52 (1999)

13. C.P. Burgess, L.E. Ibanez, F. Quevedo, Phys. Lett. B 447, 257 (1999)

14. A. Chodos, E. Poppitz, Phys. Lett. B 471, 119 (1999)

15. T. Gherghetta, M. Shaposhnikov, Phys. Rev. Lett. 85, 240 (2000)

16. W.D. Goldberger, M.B. Wise, Phys. Rev. Lett. 83, 4922 (1999)

17. P. Kanti, K.A. Olive, M. Pospelov, Phys. Lett. B 481, 386-396 (2000)

18. C. Csaki, M.L. Graesser, G.D. Kribs, Phys. Rev. D 63, 065002 (2001)

19. A. Das, T. Paul, S. SenGupta, Modulus stabilisation in a backreacted warped geometry model via Goldberger-Wise mechanism. arXiv: 1609.07787 [hep-ph]

20. W.D. Goldberger, M.B. Wise, Phys. Lett. B 475, 275-279 (2000)

21. J. Lesgourgues, L. Sorbo, Goldberger-Wise variations: Stabilizing brane models with a bulk scalar. Phys. Rev. D 69, 084010 (2004)

22. O. DeWolfe, D.Z. Freedman, S.S. Gubser, A. Karch, Phys. Rev. D 62, 046008 (2000)

23. S. Chakraborty, S. SenGupta, Eur. Phys. J. C 74(9), 3045 (2014)

24. J. Lesgourgues, S. Pastor, M. Peloso, L. Sorbo, Phys. Lett. B 489, $411(2000)$

25. H. Davoudiasl, J.L. Hewett, T.G. Rizzo, Phys. Rev. Lett. 84, 2080 (2000)

26. T.G. Rizzo, Int. J. Mod. Phys. A15, 2405-2414 (2000)

27. Y. Tang, JHEP 1208, 078 (2012)

28. H. Davoudiasl, J.L. Hewett, T.G. Rizzo, JHEP 0304, 001 (2003)
29. M.T. Arun, D. Choudhury, A. Das, S. Sengupta, Phys. Lett. B 746, 266-275 (2015)

30. ATLAS Collaboration, Phys. Lett. B 710, 538-556 (2012)

31. ATLAS Collaboration, G. Aad et al., Phys. Rev. D 90, 052005 (2014)

32. H.A. Buchdahl, General relativistic fluid spheres. Phys. Rev. 116, 1027 (1959)

33. R.M. Wald, General Relativity, 1st edn. (The University of Chicago Press, Chicago, 1984)

34. T. Padmanabhan, Gravitation: Foundations and Frontiers (Cambridge University Press, Cambridge, 2010)

35. M.K. Mak, P.N. Dobson Jr., T. Harko, Maximum mass radius ratio for compact general relativistic objects in Schwarzschildde Sitter geometry. Mod. Phys. Lett. A 15, 21532158 (2000). arXiv:gr-qc/0104031

36. H. Andreasson, C.G. Boehmer, A. Mussa, Bounds on $M / R$ for charged objects with positive cosmological constant. Class. Quant. Grav. 29, 095012 (2012). arXiv:1201.5725 [gr-qc]

37. Z. Stuchlik, Spherically symmetric static configurations of uniform density in spacetimes with a non-zero cosmological constant. Acta Phys. Slov. 50, 219228 (2000). arXiv:0803.2530 [gr-qc]

38. C.A.D. Zarro, Buchdahl limit for d-dimensional spherical solutions with a cosmological constant. Gen. Relativ. Gravit. 41, 453468 (2009)

39. J. Ponce de Leon, N. Cruz, Hydrostatic equilibrium of a perfect fluid sphere with exterior higher dimensional Schwarzschild space-time. Gen. Relativ. Gravit. 32, 12071216 (2000). arXiv:gr-qc/0207050

40. M.A. Garca-Aspeitia, L.A. Urea-Lpez, Stellar stability in braneworlds revisited. Class. Quant. Grav. 32(2), 025014 (2015). arXiv:1405.3932 [gr-qc]

41. T. Harko, M.K. Mak, Anisotropic charged fluid spheres in D spacetime dimensions. J. Math. Phys. 41, 47524764 (2000)

42. R. Goswami, S.D. Maharaj, A.M. Nzioki, Buchdahl-Bondi limit in modified gravity: packing extra effective mass in relativistic compact stars. Phys. Rev. D 92, 064002 (2015). arXiv:1506.04043 [grqc]

43. U. Das, B. Mukhopadhyay, Modified Einsteins gravity as a possible missing link between sub- and super-Chandrasekhar type Ia supernovae. JCAP 1505(05), 045 (2015). arXiv:1411.1515 [astroph.SR]

44. M. Wright, Buchdahls inequality in five dimensional GaussBonnet gravity. Gen. Relativ. Gravit. 48(7), 93 (2016). arXiv:1507.05560 [gr-qc]

45. N. Dadhich, A. Molina, A. Khugaev, Uniform density static fluid sphere in Einstein-Gauss-Bonnet gravity and its universality. Phys. Rev. D 81, 104026 (2010). arXiv:1001.3922 [gr-qc]

46. N. Dadhich, S. Chakraborty, Buchdahl compactness limit for a pure Lovelock static fluid star. Phys. Rev. D 95(6), 064059 (2017). arXiv:1606.01330 [gr-qc]

47. S.H. Hendi, G.H. Bordbar, B. Eslam Panah, S. Panahiyan, Neutron stars structure in the context of massive gravity. JCAP 1707, 004 (2017)

48. N. Dadhich, S. Hansraj, B. Chilambwe, Compact objects in pure Lovelock theory. Int. J. Mod. Phys. D 26(06), 1750056 (2016). arXiv:1607.07095 [gr-qc]

49. A. Molina, N. Dadhich, A. Khugaev, Buchdahl-Vaidya-Tikekar model for stellar interior in pure Lovelock gravity. Gen. Relativ. Gravit. 49(7), 96 (2017). arXiv:1607.06229 [gr-qc] 